\title{
A multi-criteria context-sensitive approach for social image collection summarization
}

\author{
ZAHRA RIAHI SAMANI and MOHSEN EBRAHIMI MOGHADDAM* \\ Faculty of computer Science and Engineering, Shahid Beheshti University, GC, Tehran 1983969411, Iran \\ e-mail: m_moghadam@sbu.ac.ir
}

MS received 14 June 2017; revised 2 September 2017; accepted 15 September 2017; published online 20 July 2018

\begin{abstract}
Recent increase in the number of digital photos in the content sharing and social networking websites has created an endless demand for techniques to analyze, navigate, and summarize these images. In this paper, we focus on image collection summarization. Earlier methods in image collection summarization consider representativeness and diversity criteria while recent ones also consider other criteria such as image quality, aesthetic or appeal. In this paper, we propose a multi-criteria context-sensitive approach for social image collection summarization. In the proposed method, two different sets of features are combined while each one looks at different criteria for image collection summarization: social attractiveness features and semantic features. The first feature set considers different aspects that make an image appealing such as image quality, aesthetic, and emotion to create attractiveness score for input images while the second one covers semantic content of images and assigns semantic score to them. We use social network infrastructure to identify attractiveness features and domain ontology for extracting ontology features. The final summarization is provided by integrating the attractiveness and semantic features of input images. The experimental results on a collection of human generated summaries on a set of Flickr images demonstrate the effectiveness of the proposed image collection summarization approach.
\end{abstract}

Keywords. Social images; social networks; image collection summarization; ontology and knowledge based systems.

\section{Introduction}

Due to the proliferation of digital cameras and smart phones, the size of publicly available and personal photo collections is growing. It has been reported that about 500 million images are uploaded to the internet (just considering Flickr, Facebook, Instagram and Snapchat( everyday [1]. It has created an increasing demand for image collection summarization methods, which enables users for interactive navigation and exploration of large-scale image collections.

Earlier work in image collection summarization systems considered representativeness and diversity. The summary images should be as similar as possible to the original collection and as dissimilar as possible to each other [2]. The former is called coverage and the latter is called redundancy in some literature. Recent studies propose that other criteria like quality or attractiveness are also important for the summarization. Rudinac et al [3] did a crowdsourcing experiment to find out how human users perform image collection summarization. Their study showed that images that are chosen as summary were

*For correspondence semantically similar to other images. On the other hand, they found out that other criteria like context, popularity, and visual aesthetic appeal are also important for image collection summarization.

In this paper, we propose a multi-criteria approach for image collection summarization. We propose two different meaningful sets of features: semantic features that represent content of images and attractiveness features that capture how appealing an image is. The proposed method consists of three different modules: (i) a semantic module that assigns a semantic score to each image; (ii) an attractiveness module that assigns attractiveness score to each image; (iii) the last module that creates the summary considering the information from both semantic and attractiveness modules.

We propose a context-sensitive approach for extracting semantic and attractiveness features automatically. To do so, we train models using social network activities and domain ontology and apply the trained models to extract semantic and attractiveness features according to the context of images. Final summary is created using the semantic and attractiveness scores of images.

We made ground truth human-made summaries of a set of Flickr images in the domain of cities and locations to evaluate the proposed method. The results of the proposed 
method are compared with human-made summaries. Here we address the problem of low user agreement in doing the summarization [3]. The number of common images between two human made references is reported to have a mean value equal to one [3]. We propose a summary pooling method to evaluate the result of the proposed approach. In the proposed pooling method, images which are selected more frequently by human observers and highly ranked images get heavier weight in the evaluation process. The results showed that the proposed approach performs better when compared to single-criteria image summarization systems in selecting representative photos in a collection of images.

The contribution of this paper is listed below:

- A context-sensitive multi-criteria approach is proposed for image collection summarization.

- Two meaningful sets of features are extracted automatically and applied for image collection summarization.

- A summary pooling method is proposed to overcome the low user agreement in manual summaries and compare the result of the proposed method with multiple manual reference summaries.

The rest of this paper has been organized as follows. In the next section, a literature review on image collection summarization methods is provided. Then the proposed approach is presented. Experimental results and conclusions with future work are presented subsequently.

\section{Related work}

Earlier image collection summarization methods used to have unsupervised and single-criteria approaches. They considered the goal of high representativeness and low redundancy. They are classified to visual and multi-modal summarization systems. Visual summarization systems are the category of methods that work on visual features of images and multi-modal methods work on social or web images that have side (meta) data such as textual, geo, social, or other kinds of data attached to them.

Visual image summarization systems apply two main approaches: clustering algorithms and graph centrality methods. Simon et al proposed a greedy version of k-means algorithm [2]. Their algorithm iteratively selects exemplars to optimize an objective function which maximizes the similarity of the representative set to the original set and minimizes the similarity of the representative set to itself. The method proposed by [4] uses a similar objective function and does the optimization by using simulated annealing algorithm. Clustering techniques like affinity propagation [5, 6] and spectral clustering [7] have also been applied.

Graph centrality algorithms are also used in image collection summarization. Jing and Baluja [8] use the
PageRank algorithm on a similarity graph made from visual features of images. Wang et al [9] proposed a modified version of PageRank that resulted in higher diversity. Samani and Moghaddam also make a semantic similarity graph of images and use graph centrality algorithms to do image collection summarization [10].

Social or web images have usually other modalities of data attached to them. They may contain side information like social tags, comments, geographical or temporal data. Multi-modal image summarization methods use this side information for doing the summarization.

Kennedy and Naaman [11] find representative tags and location meta data and then representative images based on the location representative tags.

Textual data is another modality of information that is used by some researchers. Qian et al [12] use clustering techniques to find representative topics from textual tags associated to images and select exemplar images from representative topics. Camargo and González [13] use nonnegative matrix factorization to find latent topics in the textual space and then use textual latent topics to perform non-negative matrix factorization in the visual space.

Graph clustering on a combination of visual and textual data is another method in summarization. For example, Yan et al [14] define two sets of nodes corresponding to images and their surrounding texts. Heterogeneous edges created based on text to image correlations while homogeneous edges created using text to text correlations. The authors use complex graph clustering and spectral co-clustering algorithm to diversify image search results. Similar methods use other graph clustering algorithms like hyper-graph partitioning [15] and affinity propagation [16].

Social meta-data such as comments or bookmarks is another source of information [17]. As an Example, Jaffe and colleagues use a combination of textual, social, temporal and location meta-data with a modified version of the Hungarian clustering algorithm [18] for image collection summarization.

Recently some supervised and multi-criteria approaches have been proposed [1, 19]. Supervised approaches are based on learning methods that minimize the difference between human-made and computer-made summaries. For example, a supervised method has been proposed in [1] to learn a mixture of submodular functions to minimize their proposed V-Rouge method. V-Rouge is defined as a distance between human-made and computer-made summaries.

Rudian et al [3] proposed one of multi-criteria, supervised and multi-modal approaches. Their approach is multimodal as it uses different side information like geo tags and textual comments of images and it can be considered as multi-criteria as it considers content and some aesthetic features of images. They use a learning method based on RankSVM to learn a summarization method based on a human-generated set of summaries.

The proposed method by Shen and Tian is another multicriteria and multi-modal approach [20]. They use a combination of time, location, semantic features, and consider 
quality of images in doing the summarization. Gaussian mixture model is applied to cluster images into different events and a photo ranking algorithm was proposed to rank photos based on their representativeness.

Table 1 shows a comparison among some of the most recent work in image collection summarization. As it can be seen in the table, the proposed method is categorized to visual image collection summarization systems as it uses images as the source of information; and it is unsupervised and multi-criteria.

Social and semantic data have been used in other areas of multimedia data such as video retrieval [21] video summarization [22]. With the recent increase in the volume of available digital photos, a wider range of applications beyond their traditional ones came to benefit from Image collection summarization; such as life logging systems [23] and Object tracking [24] in this paper, we propose a multicriteria context-sensitive approach for image collection summarization applying semantic and attractiveness data.

\section{Proposed approach}

We propose here two different sets of features in our approach. The first set contains ontology features which extract semantic content of images. These high level semantic features are defined on top of low level visual features of images. The second set consists of attractiveness features which extract different aspects that make an image appealing on social networks. Attractiveness features are defined as a combination of quality, aesthetic and emotion features on top of low level visual features of images.
Figure 1 shows an overview of the proposed method. The proposed method consists of three main modules. The first module extracts ontology features and generates a score for each image which shows the amount of (semantic) information each image covers from the whole collection. We call this module as semantic module in our future references.

Second module works on attractiveness features. It extracts attractiveness features and generates a score for each image. The score is supposed to show the amount of attractiveness of each image. We call this module as attractiveness module in our future references.

The third module is the integration and summarization module. The goal of third module is to combine the output of the first two modules and extract a unique representativeness score for each image. We apply weighted average approach for the integration module. In this way, each image gets a summarization score which is calculated by weighted average of social and semantic scores.

The proposed approach goes through two phases: training and testing. In the training phase, semantic and attractiveness modules are trained.

The idea of semantic module is to find a model to make high level semantic features from low level visual features. We use the idea of [10] with some modifications. They provide the domain ontology to the system and design a classifier that can categorize images to concepts of the domain ontology. Ontologies are the knowledge backbone for many knowledge based and intelligent systems [25, 26]. Domain ontology provides the system major concepts in the domain of images. Suppose images are related to Paris. The domain ontology provides system with major visiting

Table 1. Comparison among some recent image collection summarization methods.

\begin{tabular}{|c|c|c|c|c|}
\hline Method & Modes in use & $\begin{array}{l}\text { Supervised / } \\
\text { unsupervised }\end{array}$ & Criteria & Approach \\
\hline Latha et al [27] & Tags + images & Unsupervised & $\begin{array}{l}\text { Single- } \\
\text { criteria }\end{array}$ & Optimization \\
\hline Camargo and González [13] & Text + image & Unsupervised & $\begin{array}{l}\text { Single } \\
\text { criteria }\end{array}$ & Numeric metric optimization \\
\hline $\begin{array}{l}\text { Samani and } \\
\text { Moghaddam[10] }\end{array}$ & Image & Unsupervised & $\begin{array}{l}\text { Single- } \\
\text { criteria }\end{array}$ & Semantic + raph centrality \\
\hline Yan et al [14] & Image + text & Unsupervised & $\begin{array}{l}\text { Single- } \\
\text { criteria }\end{array}$ & Graph clustering \\
\hline Zhao et al [6] & Image & Unsupervised & $\begin{array}{l}\text { Single- } \\
\text { criteria }\end{array}$ & Clustering (affinity propagation) \\
\hline Qian et al [12] & Image + text & Unsupervised & $\begin{array}{l}\text { Single- } \\
\text { criteria }\end{array}$ & Clustering \\
\hline Fang et al [19] & Images & Supervised & $\begin{array}{l}\text { Single } \\
\text { criteria }\end{array}$ & Learning topic aspects \\
\hline Tschiatschek et al [1] & Images & Supervised & $\begin{array}{l}\text { Single } \\
\text { criteria }\end{array}$ & Learning sub modularfFunction \\
\hline Rudinac et al [3] & $\begin{array}{c}\text { Image } \\
\text { tags }\end{array}$ & Supervised & Multi-criteria & Learning RankSVM \\
\hline Shen and Tian [20] & Location + time + image & Unsupervised & Multi-criteria & Clustering \\
\hline Proposed approach & Image & Unsupervised & Multi-criteria & Combining semantic and social features \\
\hline
\end{tabular}




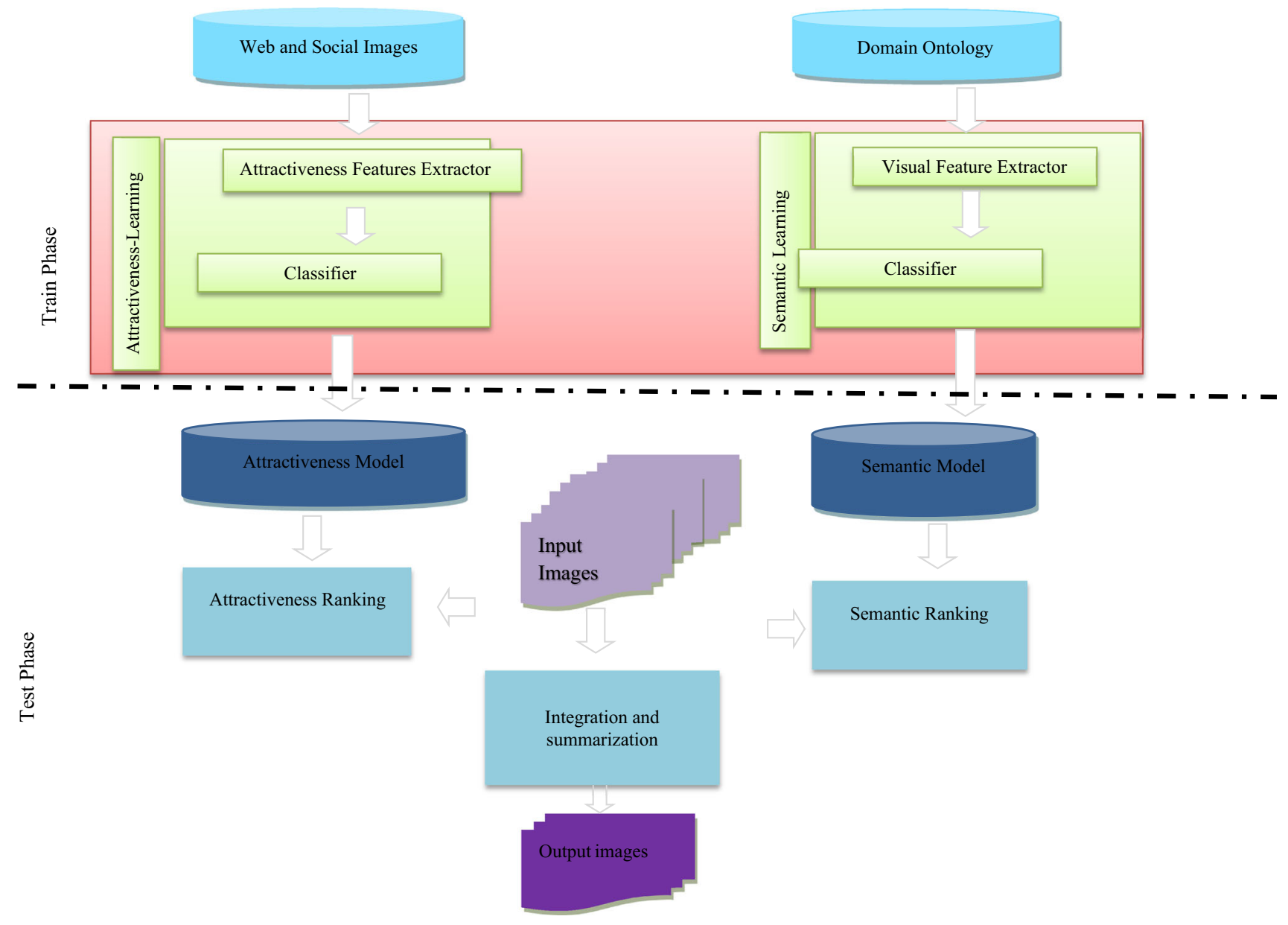

Figure 1. An overview of the proposed method.

places in Paris like Eiffel Tower and Seine River. These major visiting places are different for each city. For example, a domain ontology for Rome contains concepts like: fountain and amphitheater (corresponding to Colosseum and Trevi Fountain) and a domain ontology for Paris contains concepts like: tower and river (corresponding to Eiffel Tower and Seine River). In another word, the domain ontology provides the system the general knowledge about content and context of images. In the training phase, a classifier is trained with the concepts of the domain ontology. In the test phase, the trained classifier is applied to compute a set of ontology features. The ontology features are then used to define information content and popularity of images. Popularity is defined for all concepts of the domain ontology individually. The details of this module are presented in section 3.2.

The idea of attractiveness module is to find a model to make attractiveness features from low level visual features. We propose to train a classifier that can discriminate between social attractive and non-attractive images. In the training phase, a classifier is trained with training images of attractive versus non-attractive images. We use social behaviours of users on social networks to provide the training data for our classifier. We provide a set of attractive and non-attractive images from social images with bookmarks on them. Social behaviours vary according to the context and content of images. For example, images related to a highly-visited city like Rome may have higher range of bookmarks than a city with less number of visitors. Thus, attractiveness should be modeled differently for different contexts. In this way, we do a statistical analysis on images on various context and content individually. A set of attractive and non-attractive images are fed into the classification system based on a statistical analysis for each context. The output of the classifier in the test phase is used to make attractiveness score. The details of this module are proposed in section 3.1.

The test phase is shown in the bottom part of figure 1. In this step, the image collection is fed into the attractiveness module and semantic module. The trained attractiveness models and semantic models are used to assign semantic score and attractiveness score to each image. Finally, the integration and summarization module integrates the output of the other modules and generates the summary set. The details of this module are discussed in section 3.3.

It should be mentioned that the domain ontology and social network platform are used in the training phase and 
no side information is used in the test phase. This makes our approach applicable for images that no side information is available for them. The details of proposed modules are as follows.

\subsection{Attractiveness module}

The purpose of this module is to assign a score of attractiveness to each image. We use a classifier here that can discriminate between attractive and non-attractive images. In other word, we use a learning method that learns attractiveness features from low level visual features. The learning method gets a set of attractive and non-attractive images for each context and learns their attractiveness from their low level visual features. The classifier confidence value of each image to attractive class is used at its attractiveness score.

The first issue is to provide training samples for attractive images. We decided to use social network platform. Users in social networks can do social behaviours on images. For example, when an image is interesting to them, they may bookmark it (such as like on Facebook or favourite on Flickr); they can share it or write tags, comments or notes on it. Recent studies discuss that these meta-data can be used to determine if an image is appealing to users [28]. Social meta data have also been reported to be more important than other features such as visual or textual features [29]. Pedro and Siersdorfer [30] report that there exists a correlation between the number of bookmarks for an image and the number of other available social behaviours such as shares and comments. Thus, in this paper we decided to use social bookmarked images to provide our attractive samples. In this way, images are divided into two sets of attractive and nonattractive images according to the number of bookmarks they have.

We choose a threshold value for the number of bookmarks. In this way, images that have bookmark number greater than the threshold are considered as attractive and images with bookmark number less than the threshold are considered as non-attractive images.

The next issue is choosing the threshold value. Kardaani and Moghaddam report that the threshold value is dependent to the underlying context [28]. They show that a unique threshold value is not working for finding attractive images. They also report that social images that have bookmark counts greater than the average of the bookmarks in the corresponding context can be considered as attractive social images. Thus, we define the threshold value for each context, equal to the average of the number of bookmark of its images. Figure 2 shows a sample of attractive versus non-attractive images related to the city of Rome in our dataset.

Having two sets of attractive and non-attractive images, we use the following low level features: perceptual quality, aesthetic sensitivity and affective tune. These features are introduced in [31].

- The perceptual quality features describe the basic qualities of image, including the brightness, contrast, colourfulness, sharpness, and blur.

- The aesthetic sensitivity considers if an image is visually appealing from the aesthetics point of view. We use well-known rules, including Rule of Thirds, simplicity, and visual weights. Rule of Thirds is positioning the subject near the golden ratio points. Simplicity is simplifying the subject area comparing to image area, and visual weight is balancing the visual weight of the subject and the background.

- The affective tune features model the emotional impact of image visual elements to humans. We use line-based features and colour-based features. Line-based features are calculated according to the distributions of static and dynamic lines which reflect different emotions. The colour-based features are represented as a histogram which quantizes the impact of colour to emotions.

We use support vector machine classifiers to discriminate attractive images from non-attractive images. The classification confidence value of each image to the attractive class is used as its attractiveness score. The output of this step is the attractiveness score for different images. In the next section 3.2, the details of the semantic module are discussed.

\subsection{Semantic module}

We apply the idea of [10] with some modifications to model semantic information. Domain ontology of images is applied which contains dominant concepts of the images domain and taxonomic relations between them. In another word, domain ontology provides us the knowledge about the context of images. Figure 3 shows a sample of domain ontology related to the visiting places in Rome.

We use a classifier which categorizes images to the concepts of the domain ontology and define a set of semantic features based on the output of the classifier. Ontology features are supposed to capture semantic information in each image and are defined on top of low level visual features. The details about how to provide the domain ontology can be found in [10].

We apply a hierarchical classification technique which is proposed in [32] to do the classification among different ontology concepts. In this manner, at each semantic node in the domain ontology a classifier is applied to discriminate between immediate sub-concepts of the current node. The classification is done recursively from the root to the leaves. At each semantic node, concepts with higher confidence values are selected for further exploration and the classification is done recursively for sub-concepts. Finally, each 

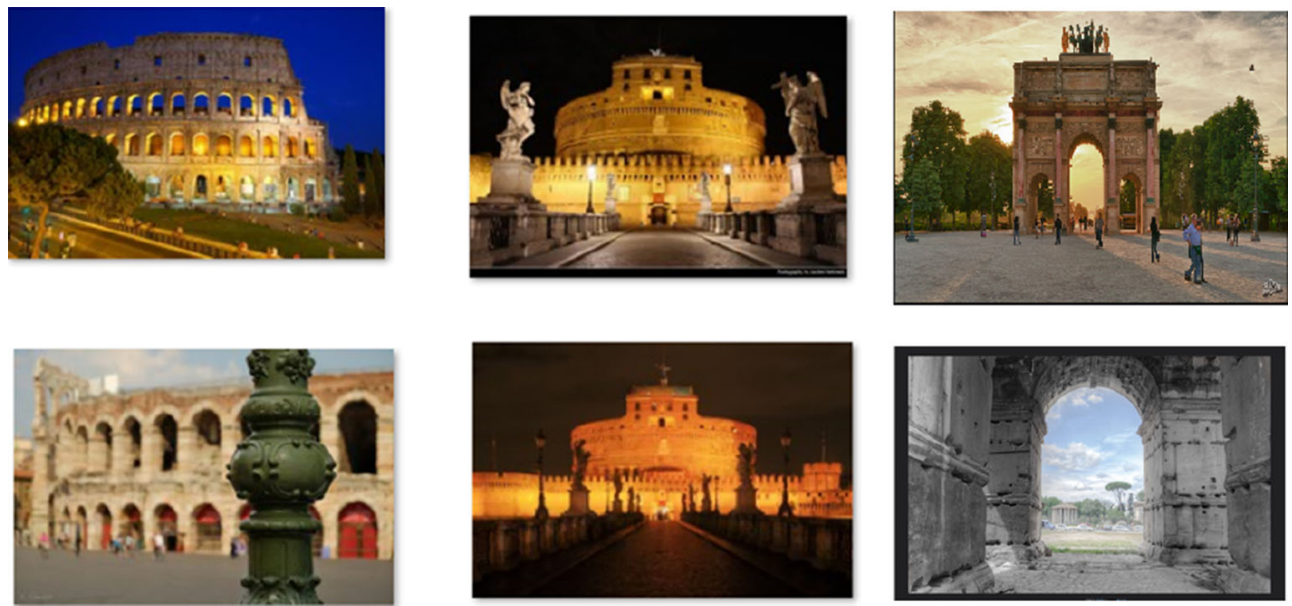

Figure 2. A sample of attractive (top) versus non-attractive (bottom) images from the city of Rome.

\section{Visiting places in Rome}

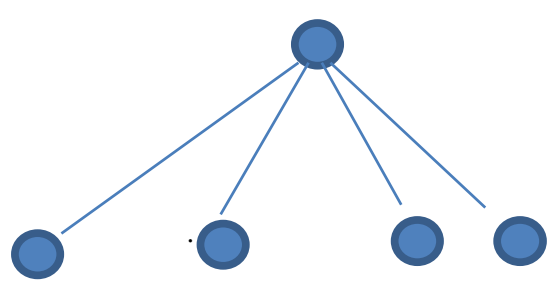

Amphitheaters

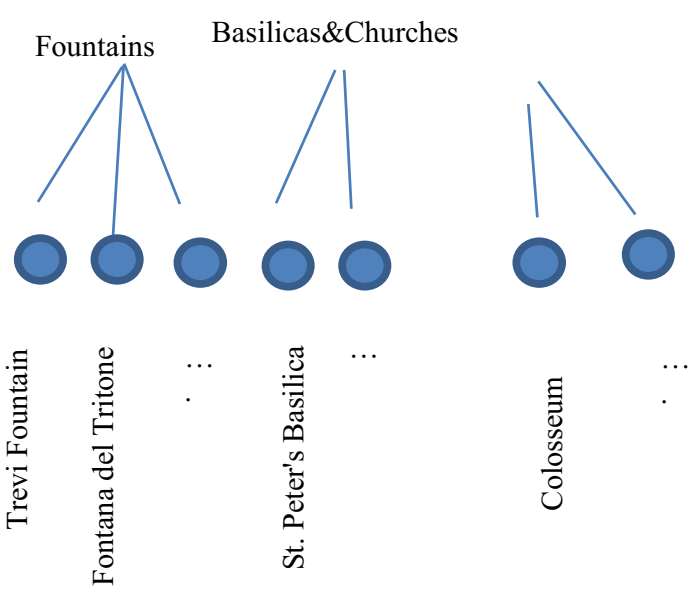

Figure 3. A domain ontology related to the city of Rome.

image is categorized to different concepts of the domain ontology. We use support vector machine classifier and Bow features defined on SIFT local features [33].

After the classification, a bag of features for each image is created by using ontology concepts:

$$
F_{i}=\left(f_{i}^{1} \ldots, f_{i}^{j}, \ldots, f_{i}^{c}\right)
$$

where $f_{i}^{j}$ is the feature of image $i$ related to concept $j$ and $c$ is the number of classes (concepts). For example, in the otology of figure 3 we have a feature vector $F_{i}$ for each image which contains different concept of the ontology e.g. fountain, Basilica and amphitheatre. $f_{i}^{j}$ is computed in the following way:

$$
f_{i}^{j}=\theta_{j} \times \operatorname{Conf}_{i}^{j}
$$

where $\operatorname{Conf}_{i}^{j}$ is the confidence value for the classification of image $i$ to class $\mathrm{j}$ and $\theta_{j}$ is computed based on equation 3:

$$
\theta_{j}=\frac{N_{j}}{N_{t}}=\frac{\sum_{i=1}^{n} f_{i}^{j}}{\sum_{j=1}^{c} \sum_{i=1}^{n} f_{i}^{j}}
$$

Based on this definition, $\theta_{j}$ is the sum of confidence values for the classification of images to class $j$ divided by the sum of confidence values for the classification of images to all classes. $\theta_{j}$ considers the popularity of a concept in computing the ontology features. Using $\theta_{j}$ gives more priority to the more frequent concepts.

As a distance measure, we use the cosine distance between image feature vectors

$$
\operatorname{Sim}_{i j}=\frac{F_{i} \cdot F_{j}}{\left|F_{i}\right|\left|F_{j}\right|}
$$

where $F_{\mathrm{i}}$ and $F_{j}$ are ontology features of the image $\mathrm{i}$ and image $\mathrm{j}$. The operator "." is a dot product between images. Although Euclidean similarity and its variations have reported to make adequate results in other areas like fingerprint matching [34] and image retrieval [35], cosine similarity makes a higher average variance in highly ranked results than Euclidean distance [36, 37]; so in this paper we use cosine distance. Finally, we define $\operatorname{Sim}_{i C}$ as its similarity to the whole collection for each image.

$$
\operatorname{Sim}_{i C}=\sum_{j=1}^{n} \operatorname{Sim}_{i j}
$$


where $n$ is the number of images in the collection. For each image i, $\operatorname{Sim}_{i C}$ shows its similarity to the whole collection from the information content point of view. Higher values of Sim for an image mean that it is more representative from information point of view. The output of this step is the semantic score for different images which is equal to $\mathrm{Sim}_{i \mathrm{C}}$ for each image.

\subsection{Integration and summarization module}

The goal of integration and summarization module is to get the output of semantic and attractive modules and do the summarization. Our idea is to use weighted average of semantic and attractiveness scores. The algorithm of summarization is shown in figure 4 . The inputs to the summarization module are the image collection and the attractiveness and semantic scores and the output is a ranked list of images in the collection while higher ordered images have higher representativeness. At the first step, the weighted average of semantic and attractiveness scores is calculated and assigned to TotalScore variable

$$
\text { TotalScore }_{j}=\frac{\sum_{i \in\{\text { Semantic, Attractiveness }\}} w_{i} \times \text { Score }_{j i}}{\sum_{i \in\{\text { Semantic }, \text { Attractiveness }\}} w_{i}}
$$

where $w_{i} s$ are the weights assigned to semantic and attractiveness scores. In this paper, we us $\left(W_{\text {Semantic }}, W_{\text {Attractiveness }}\right)=(0.53,0.47)$. These weights are found based on a user feedback experience. The first image in the summary is selected by getting the maximum value of TotalScore. In the next steps, the SemanticScore (SemScore) is updated and the weighted average is calculated iteratively.

We follow the idea of high representativeness and low redundancy for updating SemanticScore vector. By this

1. Summarization (SemScore, AttScore, C)

a. C: Original Collection

b. AttScore: The attractiveness score

c. SemScore: Semantic score (Similarity Vector)

2. For selected numbers images in the Summary

3. TotalScore $e_{j}=\frac{\sum_{i \in\{\text { Semantic, } \text { Attractiveness }\}} w_{i} \times \text { Score }_{j i}}{\sum_{i \in\{\text { Semantic, Attractiveness }\}} w_{i}}$

4. SummaryImages $=$ SummaryImage $\cup$ Image with Max (TotalScore)

5. UpdateSemanticScore (SemScore, SummaryImage)

6. End For

7. UpdateSemanticScore (SemScore, SummaryImage)

8. For each image $i$

9. SemScore $_{i}=\gamma$ SemScore $_{i^{-}}(1-\gamma)$ similarity (i, SummaryImage)

10. End Summarization

11. idea, exemplar images should be as similar as possible to the original set and as dissimilar as possible to images that are already selected. This is done in lines 7-9 (figure 4). The value of $\gamma$ does a compromise between similarity to the whole set and dissimilarity to the selected set.

According to the idea of "maximal marginal relevance" in the domain of document retrieval, there is no any wrong or correct value for $\gamma$; and it is better to start with small value of $\gamma$ (e.g. $\gamma=0.3$ ) to find out the information space in the area of query, and then to focus on more important parts using a reformulated query and a larger value of $\gamma$ (e.g. $\gamma=0.7)$. In this work, we choose $\gamma=0.8$ by experiment.

\subsection{Evaluating the proposed approach}

As a part of our evaluation, we decided to compare the results of the proposed method with human made summaries. To do so, we select 1200 images related to 12 different cities as our test set. Each city has 100 images related to it. We provide a number of users with the set of image collections and ask them to choose a small set of images (with 10 number of images) as visual summary. Users were asked to select the visual summary to capture the essence of the larger image set. Also, they were asked to order summary images based on their preferences. Finally, we have a set of manual references for each city. As an evaluation metric, we compare the results of the proposed approach with human made summaries.

At this point, an evaluation metric is required that enables us to compare the results of our approach with a set of human-made references. Rudinac et al [3] in their study discuss that there is often low user agreement between summary images. The number of common images between different summaries is reported to have the average equal to one. This will make approaches like VERT [38] which is based on comparing human-made and automatic references in the domain of video summarization hard to follow. Rudinac et al [3] propose a pyramid approach for making an optimal set of images from a set of user-made summaries. In their approach, they give higher priority to images that are chosen by more number of users. In this paper, we enhance their pyramid method and propose the following approach to combine human-made summaries. We try to take into account both the orderings provided by users and the number of users that choose an image.

We define a summary-value for each image based on the result of user orderings which is defined in the following way:

$$
\text { summary }- \text { value }_{i}=\frac{\sum_{j=1}^{N} \operatorname{order}_{j}(i)+(M-N) * C}{M}
$$

where summary value $_{i}$ is the summary-value for image $\mathrm{i}, \mathrm{N}$ is the number of time that image $\mathrm{i}$ was selected in the summary set, $\mathrm{M}$ is the total number of human-made

Figure 4. The summarization method. 


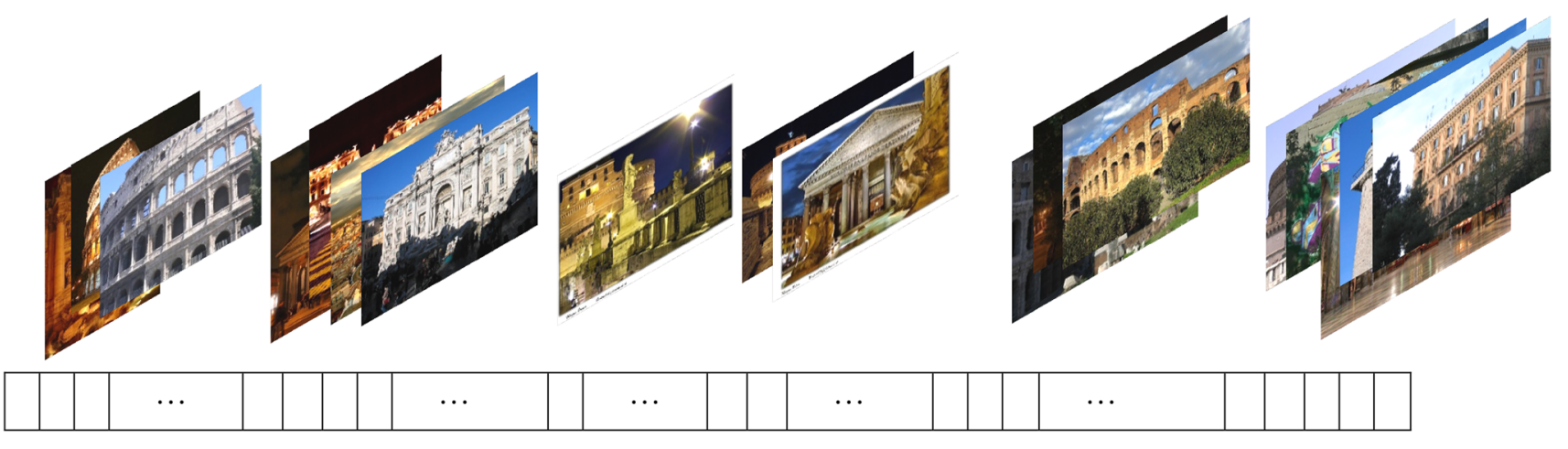

Figure 5. Summary pooling method.

references and $\mathrm{C}$ is constant number bigger than maximum possible order (in this case, any number greater than 11). The summary-value of each image is an estimation of its order by human observers considering the fact that images selected by more observers and those with lower position in the ordering should have higher impact on our the evaluation metric.

We have a list of images and their corresponding summary-value. Images are grouped according to their distinct amount of summary-value. Each distinct amount of summary-value has a group of assigned images as in figure 5 . We interpret each group as a pool of images that are of the same importance. Pools are sorted based on their increasing summary-value. The less the summary-value, the more important the pool of images.

We believe that a summary set $\mathrm{S}$ with $\mathrm{s}$ number of images that should get the maximum evaluation metric is made in the following way:

$$
S=\left\{\text { lNumber of images } \epsilon P_{p}, \bigcup_{i=1}^{p-1} \text { All images } i \in P_{i}\right\}
$$

where $P_{i}$ is ith summary pool and $\mathrm{p}$ and 1 are defined in the following way:

$$
\begin{gathered}
p=\min \left(\sum_{i=1}^{p}\left|P_{i}\right|>s\right) \\
l=s-\sum_{i=1}^{p-1}\left|P_{i}\right|
\end{gathered}
$$

We define the $\mu$ value as an evaluation metric for an automatically-generated summary $\mathrm{T}$ in the following way:

$$
\begin{gathered}
\mu=\text { Evalation metric }=\frac{1}{\mu_{S}} \sum_{i=1}^{n}(|P|-i) *\left|P_{i} \cap T\right| \\
\mu_{S}=l *\left(|P|-P_{p}\right)+\sum_{i=1}^{p-1}\left|P_{i}\right| *(|P|-i)
\end{gathered}
$$

where, $P_{i}$ is ith summary pool, $|P|$ is the number of summary pools that we have, $\mathrm{T}$ is the result of automatic image summarization system and $\left|P_{i} \cap T\right|$ is the number of common element between $P_{i}$ and T.

\section{Experimental results}

In this section, advantages of the proposed approach are demonstrated in a variety of experiments. We applied the experiments on 12000 images downloaded from Flickr website in the domain of cities and locations.

\subsection{Data set and implementation details}

The test data set contains 12000 images related to 12 cities which were downloaded from Flickr. The domain ontology about visiting places in cities was extracted from Dbpedia and ImageNet dataset was used for training the classifier with domain ontology concepts. Details can be found at [10].

We used FlickrAPI to extract and analyze the statistics of top 10000 images related to each city and find the threshold for attractiveness value. For each city, 500 attractive and 500 non-attractive images were extracted and the attractiveness classifier was trained.

Table 2. Grade assigned according to representativeness.

\begin{tabular}{llll}
\hline 0 & - & 2 & 3 \\
\hline Not representative & Low representative & Mid representative & High representative \\
\hline
\end{tabular}




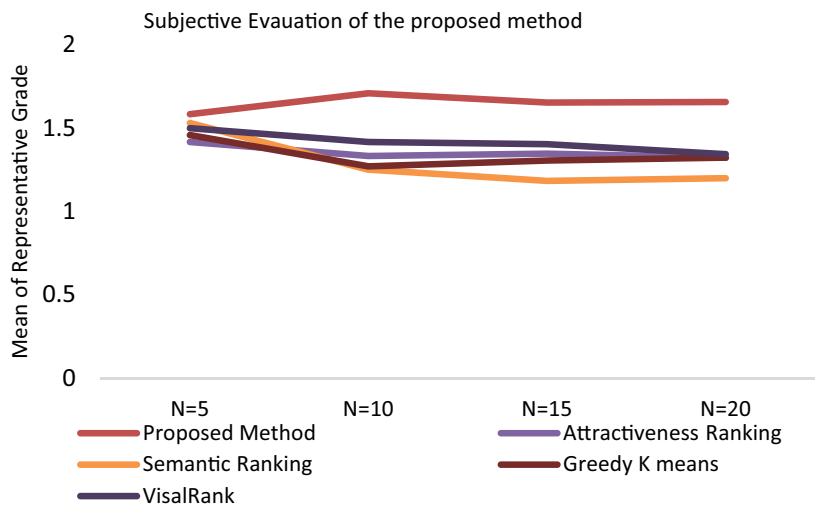

Figure 6. Subjective evaluation of the proposed method.

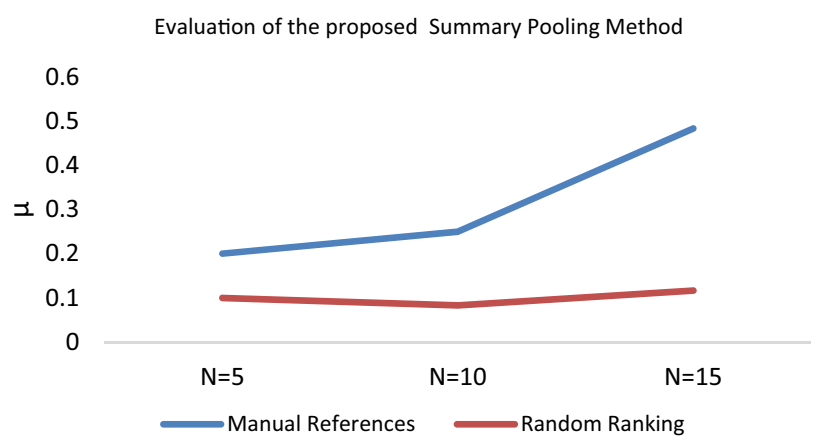

Figure 7. Evaluation of the proposed summary pooling method.

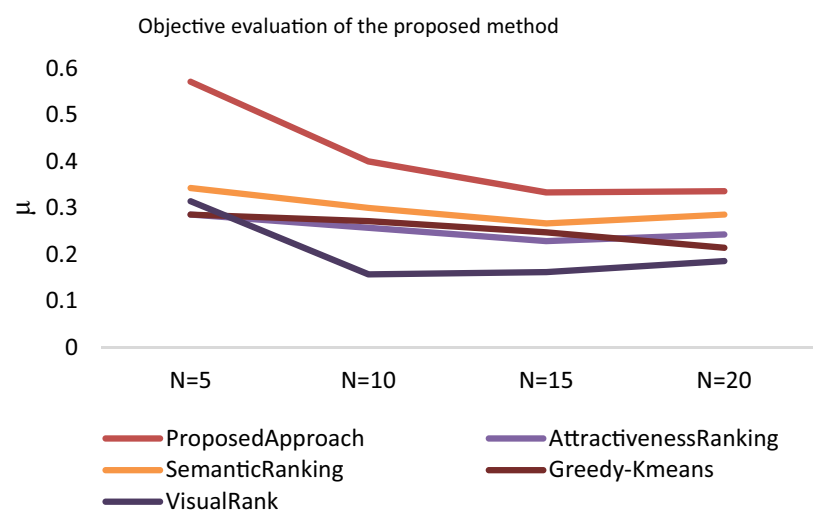

Figure 8. Objective evaluation of the proposed method.

\subsection{Method used for comparison}

We compare the proposed method with the following approaches:

- Greedy-K means: This method was proposed in [2]. They define a greedy version of k-means algorithm. The algorithm is supposed to optimize an objective function to maximize the similarity of the representative set to the original image collection and minimize the similarity of the representative set to itself.

- Visual Rank: This method was proposed in [8]. They apply PageRank algorithm on a similarity graph of images. In the similarity graph, images are nodes and edges are their visual similarity.

- Attractiveness Ranking: This approach does the ordering of images based on the output of our attractiveness module.

- Semantic Ranking: This approach does the ordering of images based on the output of our semantic module.

The first two approaches are the most common approaches in visual and unsupervised image collection summarization approaches. The next two approaches are the evaluation of attractiveness and semantic modules individually.

\subsection{Subjective evaluation of the proposed method}

We evaluate the proposed method by objective and subjective measures. Subjective evaluation is used in image summarization systems usually $[2,8,15]$. For subjective evaluation, the first 20 top ranked images were presented to 16 graduate students who have already visited and were familiar with the selected cities. The students were asked to assign representative grade to images related to each city based on table 2.

We compute the mean of subjective representative grade for different methods. The result of comparison is shown in figure 6 . The vertical axis shows the average of subjective grade among different users and horizontal axis shows summary size changing from $\mathrm{N}=5$ to $\mathrm{N}=20$ images. As it can be seen, the proposed method has better results compared to approaches of [2] and [8] and compared to semantic and attractiveness modules working individually.

\subsection{Evaluation of the summary pooling method}

In this part, we evaluate our summary pooling method. We believe that a good summarization evaluation metric should give better score for human-made summaries compared to randomly-generated summaries. We choose different manual reference summaries with varying number of images from $\mathrm{N}=5, \mathrm{~N}=10$ and $\mathrm{N}=15$. In each case, we evaluate the $\mu$ ratio for manual references in a leave-oneout manner and get their average. We compare the result of evaluation for manual references with random generated summaries (see the related work section for visual and multi-modal image collection summarization categorization). The results are shown in figure 7. As we can see, the increasing number of human-made summaries make the distance between human-made summaries and random summaries bigger. It shows that our evaluation metric works well. 

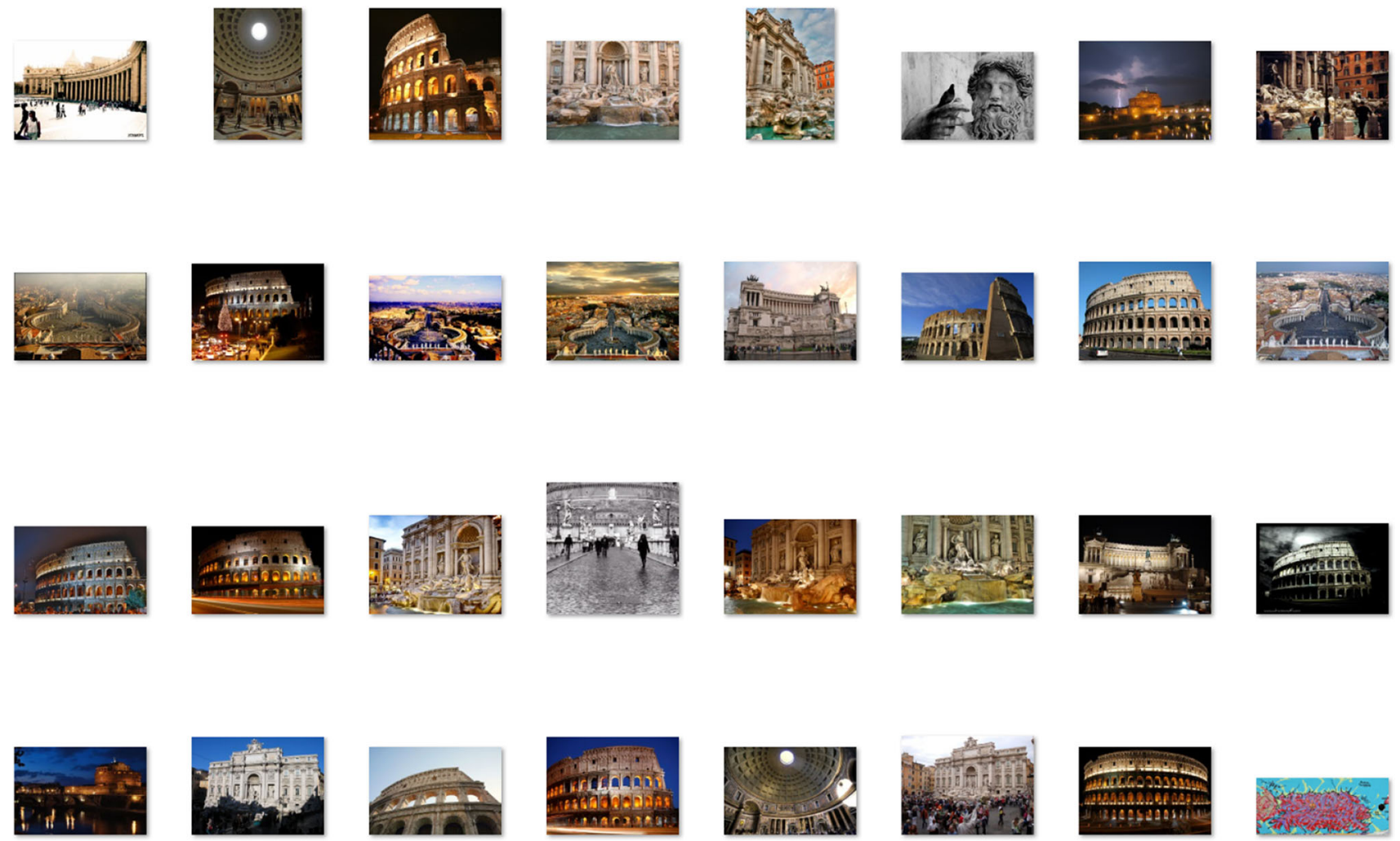

Figure 9. A set of 32 images related to the city of Rome.

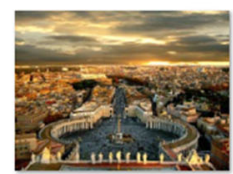

1.jpg

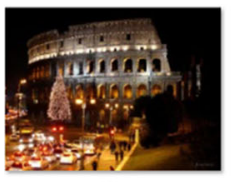

1.jpg

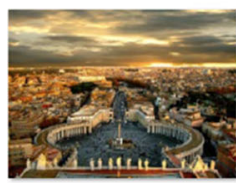

1.jpg

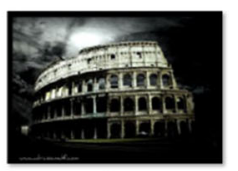

2.jpg

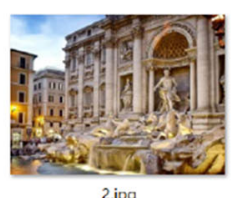

.jpg

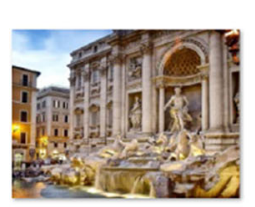

2.jpg

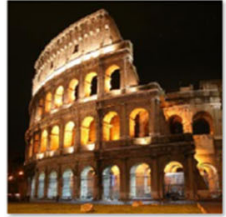

3.jpg

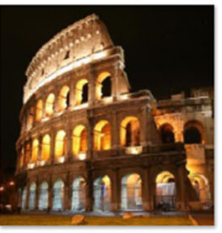

3.jpg

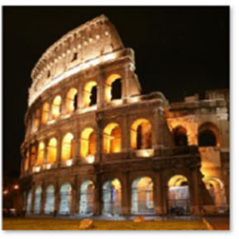

3.jpg

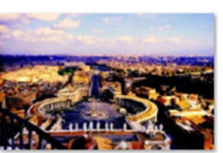

4.jpg
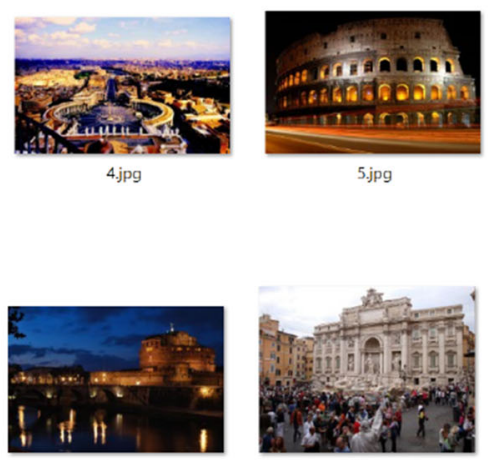

4.jpg

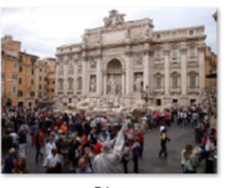

5.jpg

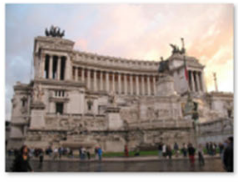

4.jpg

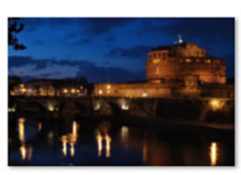

5.jpg

Figure 10. The result of summarization of images of figure 9: first 5 images with high attractiveness score (top), semantic score (middle), and the proposed approach (bottom)

\subsection{Objective evaluation of the proposed method}

For objective evaluation, a set of 1200 images, 100 images related to each city was selected from the original 12000 images. A set of human-made reference summaries was generated for each city. The original image collection was presented to 16 graduate students and they were asked to select top 10 images as the summary images. We use $\mu$ 
parameter which is defined in section 3.4 for comparing the result of the proposed method with human-made references. Figure 8 shows the result. Vertical axis is the $\mu$ parameter computed by equation 11 and horizontal axis shows different size of summaries. As it can be seen, the proposed method has better results compared to approaches of [2] and [8] and better than using semantic and attractiveness modules separately.

Figure 9 shows a set of 32 images related to Rome and figure 10 shows the result of summarization of that set with the proposed method and applying semantic and attractiveness modules alone. As it can be seen, the original set contains lots of redundant images that were related to the same aspect of Rome, while they cover different range of attractiveness. The first set contains images based on their attractiveness score and second set contains images based on their semantic score. The third set is the result of the proposed method which considers both attractiveness of images and their content.

\section{Conclusion and future work}

In this paper, a multi-criteria context-sensitive approach was proposed for social image collection summarization. We used two different modules and each one working separately on a set of features of images. We applied a context-sensitive approach to extract the features automatically. The final summary was produced by aggregating the result of these two modules.

This work has some directions to future research work. Combining ontology features with attractiveness features is done simply by the weighted average approach. There exist more advance techniques in multi-criteria ranking or rank aggregation. Applying more advance techniques for aggregation of these two metrics is an interesting idea to follow. In this method, the attractiveness model is trained on the same social network that the model is used (Flickr for both). Using different social networks as the training and test infrastructure for learning has its own challenges and is an interesting idea to follow for future work.

\section{References}

[1] Tschiatschek S, Iyer R K, Wei H and Bilmes J A 2014 Learning Mixtures of Submodular Functions for Image Collection Summarization. In: Advances in Neural Information Processing Systems, pp. 1413-1421

[2] Simon I A, Noah S and Seitz S M 2007 Scene summarization for online image collections. In: IEEE 11th International Conference on Computer Vision (ICCV 2007)

[3] Rudinac S, Larson M and Hanjalic A, 2013 Learning crowdsourced user preferences for visual summarization of image collections. IEEE Trans. Multimed. 15(6): 1231-1243
[4] Yang C, Shen J, Peng J and Fan J 2013 Image collection summarization via dictionary learning for sparse representation. Pattern Recognit. 46: 948-961

[5] Jia Y, Wang J, Zhang C and Hua X S 2008 Finding image exemplars using fast sparse affinity propagation. In: Proceedings of the 16th ACM International Conference on Multimedia

[6] Zhao Y, Hong R and Jiang J 2016 Visual summarization of image collections by fast RANSAC. Neurocomputer 172: $48-52$

[7] Crandall D J, Backstrom L, Huttenlocher C and Kleinberg J 2009 Mapping the world's photos. In: Proceedings of the 18th International Conference on World Wide Web

[8] Jing Y and Baluja S 2008 Visualrank: applying pagerank to large-scale image search. IEEE Trans. Pattern Anal. Mach. Intell. 30(11): 1877-1890

[9] Wang J, Jia L and Hua X-S 2011 Interactive browsing via diversified visual summarization for image search results. Multimed. Syst. 17(5): 379-391

[10] Samani Z R and Moghaddam M E 2016 A knowledge-based semantic approach for image collection summarization. Multimed. Tools Appl. 76(9): 11917-11939

[11] Kennedy L S and Naaman M 2008 Generating diverse and representative image search results for landmarks. In: Proceedings of the 17th International Conference on World Wide Web

[12] Qian X, Lu D, Wang Y, Zhu L, Tang Y Y and Wang M 2017 Image re-ranking based on topic diversity. IEEE Trans. Image Process. 26: 3734-3747

[13] Camargo J E and González F A 2016 Multimodal latent topic analysis for image collection summarization. Inf. Sci. 328(6): 270-287

[14] Yan Y, Liu G, Wang S, Zhang J and Zheng K 2014 Graphbased clustering and ranking for diversified image search. Multimedia Syst. 3(1): 41-52

[15] Li M Zhao C and Tang J 2013 Hybrid image summarization by hypergraph partition. Neurocomputing 119: 41-48

[16] Xu H, Wang J, Hua X S and Li S 2011 Hybrid image summarization. In: Proceedings of the 19th ACM International Conference on Multimedia

[17] Jeong J-W, Hong H-K, Heu J-U, Qasim I and Lee D-H 2012 Visual summarization of the social image collection using image attractiveness learned from social behaviors. In: IEEE International Conference on Multimedia and Expo (ICME), 2012, pp. 538-543

[18] Jaffe A, Naaman M, Tassa T and Davis M 2006 Generating summaries and visualization for large collections of geo-referenced photographs. In: Proceedings of the 8th ACM international workshop on Multimedia information retrieval

[19] Fang H, Lu W, Wu F, Zhang Y, Shang X, Shao J et al 2015 Topic aspect-oriented summarization via group selection. Neurocomputing 149: 1613-1619

[20] Shen X and Tian X 2016 Multi-modal and multi-scale photo collection summarization. Multimed. Tools Appl. 75: 2527-2541

[21] Brindha N and Visalakshi P 2017 Bridging semantic gap between high-level and low-level features in content-based video retrieval using multi-stage ESN-SVM classifier. Sādhanā 42(1): 1-10 
[22] Lu Z, Lin Y-R, Huang X, Xiong N and Fang Z 2017 Visual topic discovering, tracking and summarization from social media streams. Multimed. Tools Appl. 76(8): 10855-10879

[23] Lidon A, Bolaños M, Dimiccoli M, Radeva P, Garolera M and Giro-i-Nieto X 2017 Semantic summarization of egocentric photo stream events. In: Proceedings of the 2nd Workshop on Lifelogging Tools and Applications, pp. 3-11

[24] Osmanlığlu Y, Shakibajahromi B and Shokoufandeh A 2017 Autonomous Multi-camera Tracking Using Distributed Quadratic Optimization. In: International Workshop on Energy Minimization Methods in Computer Vision and Pattern Recognition, 2017, pp. 175-188

[25] Samani Z R and Shamsfard M 2018 The State of the Art in Developing Fuzzy Ontologies: A Survey, arXiv preprint arXiv: 1805.02290

[26] Samani Z R and Shamsfard M 2011 A fuzzy ontology model for qualitative spatial reasoning. In: 6th International Conference on Computer Sciences and Convergence Information Technology (ICCIT), 2011, pp. 1-6

[27] Latha T K K 2015 Optimization of sparse dictionary model for multimodal image summarization using firefly algorithm. Int J. Appl. Eng. Res. 10(55): 1896-1901

[28] Kardaani M and Moghadam M E 2015 Attractive social image extraction based on users' social behaviors. In: 9th Iranian Conference on Machine Vision and Image Processing (MVIP). 2015, IEEE

[29] van Zwol R, Rae A and Garcia Pueyo L 2010 Prediction of favourite photos using social, visual, and textual signals. In: Proceedings of the 18th ACM International Conference on Multimedia. ACM

[30] San Pedro J and Siersdorfer S Ranking and classifying attractiveness of photos in folksonomies. In: Proceedings of the 18th International Conference on World wide web. 2009, ACM
[31] Geng B, Yang L, Xu C, Hua X-S and Li S 2011 The role of attractiveness in web image search. In: Proceedings of the 19th ACM International Conference on Multimedia. ACM

[32] Abdollahpour Z, Samani Z R and Moghaddam M E 2015 Image classification using ontology based improved visual words. In: 2015 23rd Iranian Conference on Electrical Engineering. IEEE

[33] Chatfield K, Lempitsky V, Vedaldi A and Zisserman A 2011 The devil is in the details: an evaluation of recent feature encoding methods. In: J Hoey, M Stephen and T Emanuele (Eds) Proceedings of the British Machine Vision Conference, BMVA Press, 2011, pp. 76.1-76.12

[34] Barman S, Chattopadhyay S, Samanta D, Bag S and Show G 2014 An efficient fingerprint matching approach based on minutiae to minutiae distance using indexing with effectively lower time complexity. In: Information Technology (ICIT), 2014 International Conference on. 2014, IEEE.

[35] Malik F and Baharudin B 2013 Analysis of distance metrics in content-based image retrieval using statistical quantized histogram texture features in the DCT domain. J. King Saud Univ. Comput. Inf. Sci. 25(2): 207-218

[36] Qian G, Sural S and Pramanik S 2002 A comparative analysis of two distance measures in color image databases. In: Image Processing. 2002. Proceedings. 2002 International Conference on. 2002, IEEE

[37] Singh S, Bag S and Jenamani M 2015 Relative similarity based approach for improving aggregate recommendation diversity. In: India Conference (INDICON), 2015 Annual IEEE. IEEE

[38] Li Y and Merialdo B 2010 VERT: automatic evaluation of video summaries. In: Proceedings of the International Conference on Multimedia. ACM 